\title{
Experimental Study of Two-step Phase-shifting Digital Holography based on the Calculated Intensity of a Reference Wave
}

\author{
Jun $\mathrm{Li}^{*}$, Yang yang Pan, Jiao sheng $\mathrm{Li}$, Rong $\mathrm{Li}$, and Tao Zheng \\ School of Physics and Telecommunication Engineering, South China Normal University, \\ Guangzhou 510006, China
}

(Received March 6, 2014 : revised May 7, 2014 : accepted May 8, 2014)

\begin{abstract}
Two-step quadrature phase-shifting digital holography based on the calculated intensity of a reference wave is proposed. In the Mach-Zehnder interferometer (MZI) architecture, the method only records two quadrature-phase holograms, without reference-wave intensity or object-wave intensity measurement, to perform object recoding and reconstruction. When the reference-wave intensity is calculated from the 2D correlation coefficient (CC) method that we presented, the clear reconstruction image can be obtained by some specific algorithm. Its feasibility and validity were verified by a series of experiments with 2D objects and 3D objects. The presented method will be widely used in real-time or dynamic digital holography applications.
\end{abstract}

Keywords : Two-step phase-shifting digital holography, Phase-shifting interferometry, Computational imaging OCIS codes : (110.1758) Computational imaging; (090.0090) Holography; (100.1160) Analog optical image processing; (120.4820) Optical systems

\section{INTRODUCTION}

Digital holography is a technique that permits digital capture of holograms and subsequent reconstruction of the original object on a digital computer. It is also a convenient form for data transmission and object recognition [1, 2]. The major advantages of digital holography, easy and highly sensitive recording of holograms and quick reconstruction, have been realized by rapidly developing CCD and computer technologies. Digital holography has been applied to microscopy [3-5], interferometry [6-7], image encryption [8,9], and recognition of $3 \mathrm{D}$ objects $[10,11]$, and so on.

In recent years, two-step phase-shifting digital holography has been used in optical image reconstruction because its requires fewer hologram recording $[12,13]$. Tatsuki Taharae et.al experimentally demonstrated the parallel two-step phase-shifting digital holography which requires only the intensity distribution of the reference wave and spatial two phase-shifted holograms [14]. Jung-Ping Liu and TingChung Poon adopted a correlation method to calculate the reference wave intensity [15]. These methods are more efficient than three or four-step phase-shifting holography, but they still need to record additionally the reference wave intensity or the object wave intensity. In this paper, we proposed an image reconstruction method with two-step quadrature phase-shifting digital holography, in which there is no need to record the reference wave intensity and the object wave intensity. In the system only two holograms are required to reconstruct the original object so it is more suitable for a real-time situation. We performed experiments to verify that our method can be used in $2 \mathrm{D}$ and $3 \mathrm{D}$ objects holography and there will not be convergence errors in CC calculation. We have applied this method well to holographic compressive imaging [16]. Section 2 introduced the principles of our method and Section 3 demonstrated the experimental results.

\section{FUNDAMENTAL PRINCIPLES}

\subsection{Hologram Recording}

A typical quadrature phase-shifting holographic setup is shown in Fig. 1., where a linearly polarized laser beam is expanded, collimated, and then divided into an object

\footnotetext{
*Corresponding author: lijunc@126.com

Color versions of one or more of the figures in this paper are available online.
} 


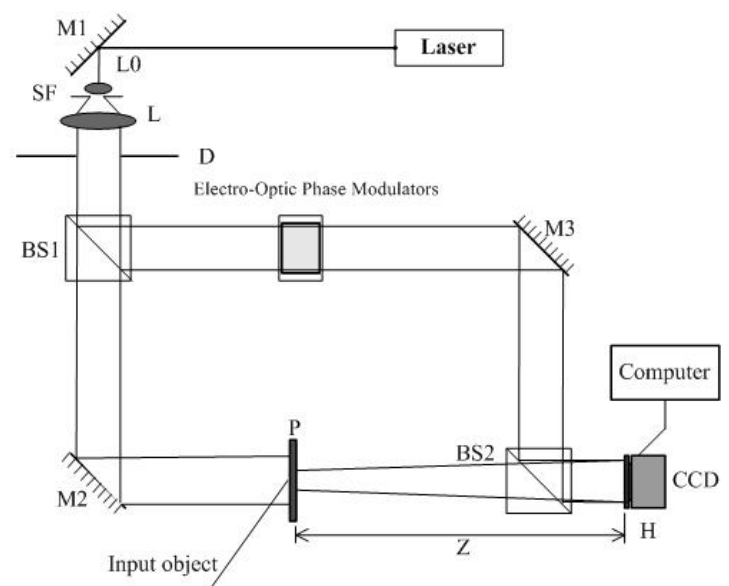

FIG. 1. Setup of image recording with two-step-only quadrature phase-shifting digital holography.

beam and a reference beam. The object beam illuminates plane $\mathrm{P}$, where the original image $\mathrm{O}$ is put on the plane $\mathrm{P}$. The phase of the reference wave is controlled by an electro-optic phase modulator. Then the two waves overlap to form interferograms on the $\mathrm{CCD}$ plane $\mathrm{H}$.

Let us assume that the plane reference wave is simply given by the real amplitude $A_{r}$, and a complex object field in the plane $\mathrm{P}$ is $O\left(x_{0}, y_{0}\right)$, the complex object field in the recording plane $\mathrm{H}$ is $u_{H}\left(x_{H}, y_{H}\right)$. The distance between planes $\mathrm{P}$ and $\mathrm{H}$ is $Z$. Based on the traditional two-step quadrature phase-shifting holography [12, 13], when we regulate the electro-optic phase modulator and set the phases of the reference wave in the first and second exposure to 0 and $\pi / 2$, respectively, then two on-axis quadrature-phase holograms $I_{H I}$ and $I_{H 2}$ in the CCD plane $\mathrm{H}$ are recorded sequentially and expressed as

$$
\begin{aligned}
I_{H 1}\left(x_{H}, y_{H}\right) & =\left|u_{r 1}+u_{H}\right|^{2} \\
& =I_{0}\left(x_{H}, \quad \mathrm{y}_{H}\right)+2 \operatorname{Re}\left[u_{H}\left(x_{H}, y_{H}\right)\right] * A_{r}, \\
I_{H 2}\left(x_{H}, y_{H}\right) & =\left|u_{r 2}+u_{H}\right|^{2} \\
& =\left|i A_{r}+u_{H}\right|^{2} \\
& =I_{0}\left(x_{H}, y_{H}\right)+2 \operatorname{Im}\left[u_{H}\left(x_{H}, y_{H}\right)\right] * A_{r},
\end{aligned}
$$

where $I_{0}$ is the zero-order light given by

$$
I_{0}\left(x_{H}, y_{H}\right)=A_{r}^{2}+\left|u_{H}\right|^{2}
$$

Only with the two recorded interferograms $I_{H 1}$ and $I_{H 2}$, we can reconstruct the original object. In the process, neither the reference wave intensity nor the object wave intensity needs to be recorded for reconstruction of the original image.

\subsection{Hologram Reconstruction}

According to above image recording method, if the reference wave intensity $A_{r}^{2}$ and the object wave intensity $\left|u_{H}\right|^{2}$ are measured, the original image can be reconstructed correctly with the elimination of the twin-image noise and the zero-order light. We show that such a goal can be achieved from an algorithm, which determines the reference wave intensity $A_{r}^{2}$ directly from the hologram without the actual need to record the reference wave intensity at all.

We find the reference wave intensity by searching all of the most possible values. From holographic imaging principle, $A_{r}^{2}$ should be within 0 and the maximum of $I_{H l}$, we then construct a parameter called the 2D correlation coefficient to evaluate the correlation of the reconstruction results by single hologram and two-step phase-shifting holograms. Single hologram contains zero-order light and twin image, but based on the two-step phase-shifting holography method, two-step phase-shifting holograms can restrain zero-order light and twin image [12,13]. So if the reference wave intensity we determined is correct, there will be just one minimum point in the $\mathrm{CC}$ curve. Consequently, we can reconstruct the original image without convergence errors.

$$
C C=a b s\left(E_{T}\right) \otimes a b s(E),
$$

Where abs $(E)$ denotes the absolute value of $(E) ; E_{T}$ is the image reconstructed by $I_{H l}$; $E$ denotes the reconstructed image at different intensity values of the reference wave $A_{r}^{2}$ (the value is gotten by searching from 0 to $\max \left(I_{H I}\right)$ without actual measurements) with two holograms $I_{H 1}, I_{H 2}$, which is calculated by Eqs. (9)-(10) with the assumed values $A_{r c}{ }^{2}$ of the reference wave $A_{r}{ }^{2}, \otimes$ denotes the 2D correlation operation.

We then draw the curve of $\mathrm{CC}$ versus $A_{r \mathrm{c}}$ and set the criterion such that the minimum point of the curve locates the actual value of $A_{r}^{2}$ (see Fig. 2(c)). With this found value of $A_{r}^{2}$ we can reconstruct the original image based on Eqs. (9)-(11).

Now in this way only two quadrature-phase holograms are needed to reconstruct the original image without zeroorder image and twin-image, and the clear reconstructed image can be obtained at high speed by a certain algorithm.

The algorithm can be expressed as follows:

Replace the $A_{r}$ in Eqs. (1)-(3) with the value $A_{r c}$ found in the above method, so we can construct a complex hologram $I_{C}$ according to

$$
\begin{aligned}
I_{C} & =I_{H 1}+i I_{H 2} \\
& =I_{0}+i I_{0}+2 A_{r c}\left[\operatorname{Re}\left(u_{H}\right)+i \operatorname{Im}\left(u_{H}\right)\right] \\
& =I_{0}+i I_{O}+2 A_{r c} u_{H}
\end{aligned}
$$

Where $u_{H}$ is the complex amplitude of the object light on the CCD camera, which is given by $u_{H}=\operatorname{Re}\left(u_{H}\right)+i \operatorname{Im}\left(u_{H}\right)$. By taking the square of the absolute value of both sides of 
Eq. (5), we obtain the solution to a quadratic equation in $I_{0}$.

$$
I_{0}=\frac{2 A_{r c}{ }^{2}+I_{H 1}+I_{H 2}}{2} \pm \frac{\left[\left(2 A_{r c}{ }^{2}+I_{H 1}+I_{H 2}\right)^{2}-2\left(I_{H 1}{ }^{2}+I_{H 2}{ }^{2}+4 A_{r c}{ }^{2}\right)\right]^{1 / 2}}{2}
$$

Moreover, from Eqs. (1)-(2), we calculate

$$
2 A_{r c}{ }^{2}+I_{H 1}+I_{H 2}=2 I_{0}+2\left[A_{r c}+\operatorname{Re}\left(u_{H}\right)+\operatorname{Im}\left(u_{H}\right)\right] A_{r c},
$$

from which, we can write

$$
I_{0}=\frac{2 A_{r c}{ }^{2}+I_{H 1}+I_{H 2}}{2}-\left[A_{r c}+\operatorname{Re}\left(u_{H}\right)+\operatorname{Im}\left(u_{H}\right)\right] A_{r c},
$$

Comparing Eq. (6) with Eq. (8), we find that if $A_{r c}+$ $\operatorname{Re}\left(u_{H}\right)+\operatorname{Im}\left(u_{H}\right)>0$, the minus sign should be chosen for Eq. (6). The quantity of $A_{r c}+\operatorname{Re}\left(u_{H}\right)+\operatorname{Im}\left(u_{H}\right)$ will be positive everywhere if the intensity of reference wave is larger than that of the object wave intensity in the $\mathrm{CCD}$ plane, which is generally true in practice $[12,13]$. This will ensure that Eq. (6) takes the minus sign and then we can calculate

$$
\begin{aligned}
I_{0} & =\frac{2 A_{r c}{ }^{2}+I_{H 1}+I_{H 2}}{2} \\
& -\frac{\left[\left(2 A_{r c}{ }^{2}+I_{H 1}+I_{H 2}\right)^{2}-2\left(I_{H 1}{ }^{2}+I_{H 2}{ }^{2}+4 A_{r c}{ }^{2}\right)\right]^{1 / 2}}{2},
\end{aligned}
$$

Then from Eq. (5) we can calculate the complex amplitude in the CCD plane

$$
u_{H}=\frac{\left(I_{H 1}-I_{0}\right)+i\left(I_{H 2}-I_{0}\right)}{2 A_{r c}},
$$

According to Eqs. (9)-(10), we can obtain zero-orderand twin-image-free hologram $u_{H}\left(x_{H}, y_{H}\right)$ with the two captured quadrature-phase interferograms and the reference wave intensity acquired with above the $\mathrm{CC}$ method. Once $u_{H}\left(x_{H}, y_{H}\right)$ is known, we can retrieve the complex field in the original object plane $\mathrm{P}$ as

$$
O^{\prime}\left(x_{0}, y_{0}\right)=\operatorname{IFR}_{Z}\left\{u_{H}\left(x_{H}, y_{H}\right)\right\},
$$

Where $I F R_{Z}$ denotes the inverse Fresnel transformation of distance $Z$. That is to say, original images have been well reconstructed.

Previously, Liu et al. [15] proposed a seemingly similar correlation method to calculate the reference wave intensity. However, first they defined a different amplitude ratio A from us as

$$
\mathrm{A} \equiv \frac{R}{1 / 2[\max (|\vartheta|)+\min (|\vartheta|)]},
$$

where $R$ is the amplitude of the reference light on the CCD plane and $\vartheta$ denotes the complex amplitude of the object light on the CCD plane, such that when $\mathrm{A} \geq 1$, only two quadrature phase holograms are needed to obtain a twin-image- and zero-order-free object wave in their method. However, according to the equation, the value of $\mathrm{A}$ is related to object wave intensity on the CCD plane and diffraction distance, so in order to reconstruct different original images or dynamic scenes, different object wave intensity on the CCD plane is required to be recorded to judge whether $A \geq 1$. In contrast, in our method, we just need to evaluate the amplitude ratio of reference wave intensity and illumination light intensity in the light path. Based on CC calculation, when the amplitude ratio of reference wave intensity and illumination light intensity in the light path is larger than 1.329 , the correlation coefficient of the reconstruction object and the original object is 1 , thus we can reconstruct original images accurately. So our method can reach the goal to record only two quadrature phase holograms. Secondly, the correlation coefficient defined in their paper, $\mathrm{NCP} \equiv \frac{\operatorname{real}\left\{\left[E_{T} \otimes E_{R}\right]_{\max }\right\}}{\left[E_{R} \otimes E_{R}\right]_{\max }}$ is obviously different from ours.

\section{EXPERIMENTAL RESULTS}

A series of experiments has been made to verify the feasibility of our proposed method. We shall now show a series of results based on the following conditions. The wavelength of the He-Ne laser is $632.8 \mathrm{~nm}$. The quantitative bit depth of the MVC-1000 type CCD is 8 bit and the largest number of pixels is $1280(\mathrm{H})$ by $1024(\mathrm{~V})$ with pixel size of $5.2 \mu \mathrm{m} \times 5.2 \mu \mathrm{m}$. The quantitative bit depth of the Pike F-421 type CCD is 14 bit and the largest number of pixels is $2048(\mathrm{H})$ by $2048(\mathrm{~V})$ with pixel size of $7.4 \mu \mathrm{m} \times 7.4 \mu \mathrm{m}$. The NEW FOCUS $4002 \mathrm{M}$ type electro-optic phase modulator is controlled by a voltage amplifier and a lag wave plate is used for phase modulation.

First we verify the validity of this method by $2 \mathrm{D}$ objects reconstruction experiments. The object we use is the transmission type object "SCNU", as shown in Fig. 2(a) with the size of $1.25 \mathrm{~cm} \times 1.25 \mathrm{~cm}$. The parameter that we use is $d=46.1 \mathrm{~cm}$. We regulate the electro-optic phase modulator and set the phases of the reference wave in the first and second exposure to 0 and $\pi / 2$, respectively. We get two quadrature-phase interferograms on the CCD plane, one interferogram $I_{H l}$ is shown in Fig. 2(b). Based on the idea of CC discussed previously, Fig. 2(c) shows the CC curve for different $A_{r c}$ values. From the figure, if the amplitude of the reference light is smaller than 0.34 , there 


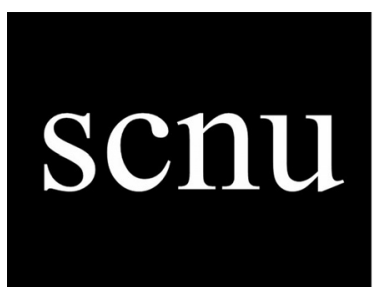

(a)

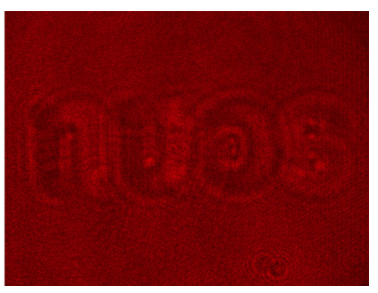

(b)

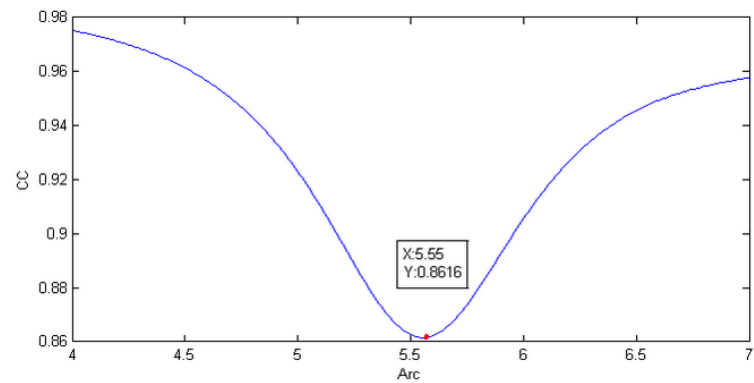

(c)

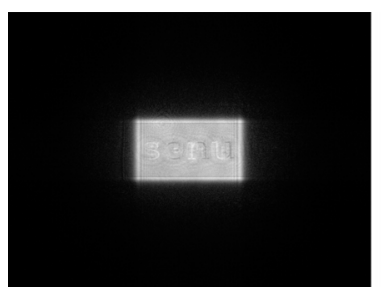

(d)

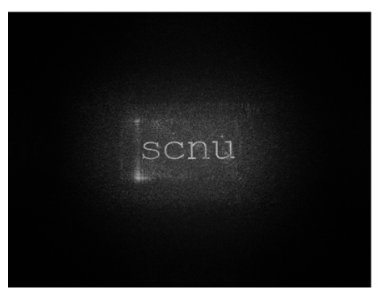

(f)

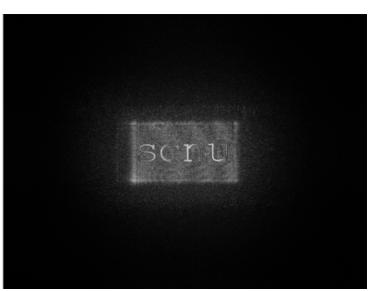

(e)

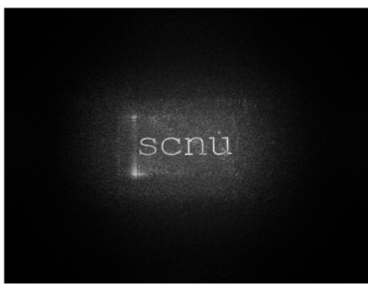

(g)
FIG. 2. The experimental results of two-step algorithm based on the calculated intensity of reference beam for $2 \mathrm{D}$ objects: (a) original image, (b) one of the two holograms, (c) the curve of correlation coefficient versus reference wave amplitude Arc, (d) reconstruction result of single hologram, (e) reconstruction result using our method, (f) minus the mean value of $(\mathrm{e}),(\mathrm{g})$ mean filtering of $(\mathrm{f})$.

will exist convergence errors. However, this situation will not appear in the experiments. With the increase of the reference wave amplitude, there will be just one minimum point in the $\mathrm{CC}$ curve. And the true amplitude of the reference wave is 5.55. Fig. 2(e) shows the reconstruction by using the value found in the $\mathrm{CC}$ method corresponding to those of Fig. 2(c). Reconstruction results of single hologram and reconstruction results by subtracting the mean value and mean filtering are shown in Fig. 2(d), (f) and (g). Compared with Fig. 2(d), Fig. 2(e) using our method shows a zero-order-and twin-image-free reconstruction image

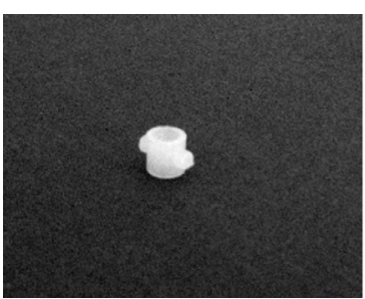

(a)

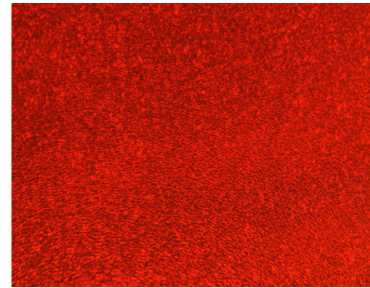

(b)

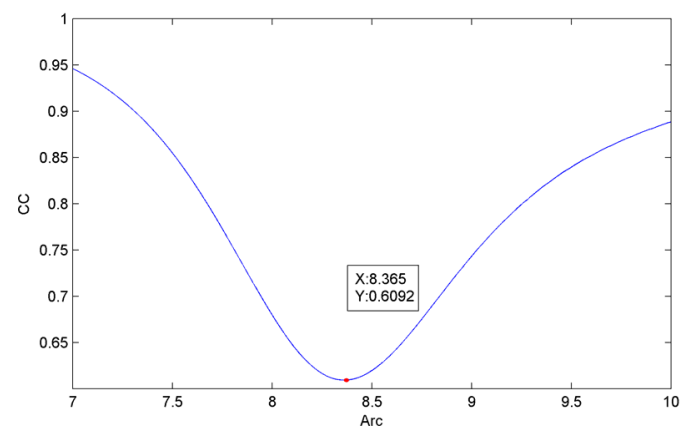

(c)

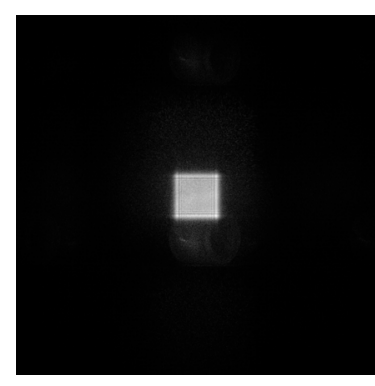

(d)

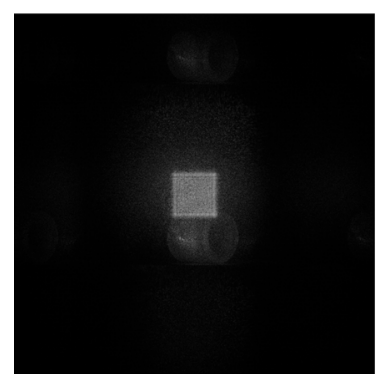

(f)

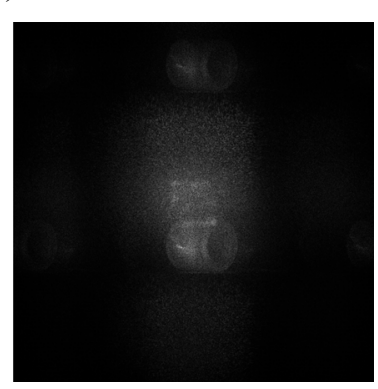

(e)

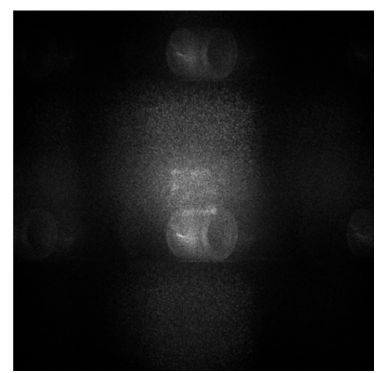

(g)
FIG. 3. The experimental results of two-step algorithm based on the calculated intensity of reference beam for 3D objects: (a) small plastic ring using as 3-D object, (b) one of the two holograms, (c) the curve of correlation coefficient versus reference wave amplitude Arc, (d) reconstruction result of single hologram, (e) reconstruction result using our method, (f) reconstruction result using four-step phase-shifting holography, (g) mean filtering of (e).

and we can get a clear reconstruction result by subtracting the mean value (see Fig. 2(f)) and mean filtering (see Fig. 2(g)).

Then, the $3 \mathrm{D}$ objects reconstruction has been tested. The object we use in this experiment is a plastic ring is as shown in Fig. 3(a) with size of $6 \mathrm{~mm} \times 6 \mathrm{~mm} \times 6 \mathrm{~mm}$. The 
parameter is $d=34.7 \mathrm{~cm}$. We regulate the electro-optic phase modulator for $3 \mathrm{D}$ objects. According to $2 \mathrm{D}$ method, the true value of the reference wave is 8.37 (see Fig. 3(c)). Finally, we obtain the reconstruction image, as shown in Fig. 3(e). Reconstruction results of single hologram and reconstruction result by subtracting the mean filtering are shown in Fig. 3(d) and (g). From the pictures we can see that we can also reconstruct the $3 \mathrm{D}$ original object with zero-order-and twin-image-free.

To further investigate the effectiveness of our method, we reconstruct the object by four-step phase-shifting holography, as shown in Fig. 3(f). Due to the limitation of our experimental instrument, the experimental result for four-step phase-shifting digital holography is not ideal. However, with this limitation, the reconstruction result by the two-step phase-shifting holography method is better than the reconstruction result by four-step phase-shifting holography. Therefore, in the low-level optical equipment conditions, two-step phase-shifting holography can work better than four-step phase-shifting holography. And our method will also get ideal results in the high precision equipment conditions as well as in four-step phase-shifting holography. So the method proposed in this paper is effective not only in the high precision equipment conditions but also in the low-level optical equipment conditions. The results of the experiments show that our method not only can be applied to 2D object reconstruction, but also can be applied to 3D object reconstruction.

\section{CONCLUSIONS}

In this paper the two-step phase-shifting digital holography based on the calculated intensity of reference wave algorithm is proposed. With only two captured quadrature-phase holograms known, in addition to the acquired reference-wave intensity, which is not directly recorded in advance but calculated from CC method with two holograms, the clear retrieved image can be reconstructed at high speed. We can obtain clear reconstruction images without reference-wave intensity or object-wave intensity measurement. In the traditional two-step phase-shifting digital holography method, object wave intensity and the reference wave intensity need to be recorded to reconstruct the original image. Due to the high storage requirement of image data and long recording time, these methods have some difficulty particularly in real-time dynamic measurement. Compared with traditional two-step phase-shifting digital holography, our method just need to calculate the reference wave intensity by the $\mathrm{CC}$ method instead of recording object wave intensity and reference wave intensity. In this method, our system need to record only two quadrature phase holograms and we don't need to record other data on the CCD plane. It greatly reduces the recording time. For real-time dynamic measurement, we just need to modulate the phase rapidly by phase shifter and get synchronization measurement on the $\mathrm{CCD}$ plane to achieve real-time dynamic imaging. In addition, our method can also be used in one-step parallel phase-shifting digital holography to simplify the system. So, this proposed method can improve the speed of holographic imaging and can be considered as a real-time solution. For 3D objects, two-step phase-shifting holography works better than four-step phase-shifting holography. It reduces by half the storage requirements of the digital hologram. The results of the experiments show that the algorithm we proposed is effective. We believe that the method proposed in this paper will be widely used in parallel holography [17,18], and will provide an effective solution to the real-time and dynamic holographic field.

\section{ACKNOWLEDGMENT}

This study was supported by the Project of Department of Education of Guangdong Province, China (No.2013 KJCX 0058).

\section{REFERENCES}

1. T. S. Huang, "Digital holography," Proc. IEEE 59, 13351346 (1971).

2. W. Jueptner and U. Schnars, Digital Holography: Digital Hologram Recording, Numerical Reconstruction, and Related Techniques (Springer Berlin Heidelberg, Berlin, Germany, 2005), Chapter 3.

3. C. Depeursinge, "Digital holography applied to microscopy," in Digital Holography and Three-Dimensional Display (Springer US, New York, USA, 2006), pp. 95-143.

4. T. Tahara, K. Ito, T. Kakue, M. Fujii, Y. Shimozato, Y. Awatsuji, K. Nishio, S. Ura, T. Kubota, and O. Matoba, "Parallel phase-shifting digital holographic microscopy," Biomedical Optics Express 1, 610-616 (2010).

5. D. Weigel, T. Elsmann, H. Babovsky, A. Kiessling, and R. Kowarschik, "Combination of the resolution enhancing image inversion microscopy with digital holography," Opt. Commun. 291, 110-115 (2013).

6. L. Cai, X. Meng, Y. Wang, X. Shen, G. Dong, and X. Yang, "Virtual shearing interferometry by digital holography," Opt. Commun. 259, 64-69 (2006).

7. T. Kreis, "Holographic Interferometry," in Handbook of Holographic Interferometry: Optical and Digital Methods (Wiley, 2005), pp. 35-219.

8. S. K. Gil, S. H. Jeon, N. Kim, and J. R. Jeong, "Successive encryption and transmission with phase-shifting digital holography," in Integrated Optoelectronic Devices, Proc. SPIE (California, USA, 2006), vol. 6136, pp. 613615-1 613615-8.

9. E. Tajahuerce and B. Javidi, "Encrypting three-dimensional information with digital holography," Appl. Opt. 39, 6595$6601(2000)$.

10. P. L. Carmona, B. Javidi, F. Pla, and E. Tajahuerce, "Photon counting 3D object recognition using digital holography," Photonics Journal 5, 6900309 (2013). 
11. B. Javidi and E. Tajahuerce, "Three-dimensional object recognition by use of digital holography," Opt. Lett. 25, 610-612 (2000).

12. P. Y. Guo and A. J. Devaney, "Digital microscopy using phase-shifting digital holography with two reference waves," Opt. Lett. 29, 857-859 (2004).

13. X. Meng, L. Cai, X. Xu, X. Yang, X. Shen, G. Dong, and Y. Wang, "Two-step phase-shifting interferometry and its application in image encryption," Opt. Lett. 31, 1414-1416 (2006).

14. T. Tahara, K. Ito, M. Fujii, T. Kakue, and Y. shimozato, "Experimental demonstration of parallel two-step phaseshifting digital holography," Opt. Express 18, 18975-18980
(2010).

15. J. P. Liu and T. C. Poon, "Two-step-only quadrature phaseshifting digital holography," Opt. Lett. 34, 250-252 (2009).

16. J. Li, Y. Li, Y. Wang, and K. Li, "Two-step holographic imaging method based on single-pixel compressive imaging," J. Opt. Soc. Korea 18, 146-150 (2014).

17. N. T. Shaked, T. M. Newpher, M. D. Ehlers, and A. Wax, "Parallel on-axis holographic phase microscopy of biological cells and unicellular microorganism dynamics," Appl. Opt. 49, 2872-2878 (2010).

18. Y. Awatsuji, T. Tahara, A. Kaneko, T. Koyama, K. Nishio, S. Ura, T. Kubota, and O. Matoba, "Parallel two-step phaseshifting digital holography," Appl. Opt. 47, 183-189 (2008). 